\title{
Estudio de validación del International Affective Picture System en niños y adolescentes argentinos
}

\author{
A Validation Study of The International Affective Picture System in Children and \\ Adolescents in Argentina \\ Leonel Mina, Liliana Bakker, Josefina Rubiales, \& Rocío González \\ Universidad Nacional de Mar del Plata, Mar del Plata, Argentina \\ Consejo Nacional de Investigaciones Científicas y Técnicas (Conicet), Mar del Plata, Argentina
}

El objetivo del estudio fue analizar el comportamiento psicométrico del conjunto de imágenes para niños y adolescentes del International Affective Picture System. La muestra estuvo compuesta por 141 niños y adolescentes de entre 7 y 14 años, divididos en tres franjas etarias que evaluaron las 60 imágenes en las dimensiones de valencia y arousal. Los resultados mostraron una baja activación para las imágenes consideradas agradables y alta activación para las imágenes consideradas desagradables, con una correlación fuerte y negativa para las segundas. La distribución de las imágenes en el espacio afectivo evidenció una mayor dispersión y menor inclinación de la activación para las imágenes agradables respecto de las desagradables. El comportamiento de la dimensión de valencia sostiene a esta como el eje principal sobre la cual se organizan las emociones, la cual está vinculada con el proceso de adaptación y evolución humana, a diferencia de la dimensión de arousal que estaría más influenciada por factores socioculturales y de aprendizaje. La distribución de los estímulos afectivos en el espacio bidimensional refleja la organización de las emociones para niños y adolescentes argentinos, demostrándose que el IAPS puede ser un instrumento fiable para evaluar emociones en esta población.

Palabras clave: IAPS, emoción, niños, adolescentes.

The aim of the study was to analyze the psychometric behavior of the set of images for children and adolescents of the International Affective Picture System. The sample consisted of 141 children and adolescents between 7 and 14 years old, divided into three age ranges, that evaluated 60 images in the valence and arousal dimensions. The results showed a low activation for images considered as pleasant and high activation with a negative strong correlation for images considered as unpleasant. The distribution of the images in the affective space showed a greater dispersion and less inclination activation for pleasant pictures, compared to unpleasant. The valence dimension consists in the main axis that emotions are organized, and it is related to the process of human adaptation and evolution, compared with the arousal dimension that would be more influenced by learning and sociocultural factors. The distribution of affective stimuli in two-dimensional space reflects the organization of emotions for children and adolescents in Argentina, demonstrating that IAPS may be a reliable instrument to assess emotions in this population.

Keywords: IAPS, emotion, children, adolescents.

Agradecemos a Peter J. Lang y a Margaret M. Bradley el generoso aporte de las imágenes de los estímulos afectivos del IAPS.

Contacto: L. Mina. Funes 3280, Cuerpo V, Nivel III, Mar del Plata (7600), Argentina. Correo electrónico: mina.leonel@yahoo.com.ar

Cómo citar: Mina, L., Bakker, L., Rubiales, J., \& González, R. (2017). Estudio de validación del International Affective Picture System en niños y adolescentes argentinos. Revista de Psicología, 26(2), 1-16.

http://dx.doi.org/10.5354/0719-0581.2017.47953 


\section{Introducción}

La investigación psicológica en el ámbito de la emoción ha experimentado un notable crecimiento en los últimos treinta años (Moltó Brotons et al., 2013). Si bien los intentos para definir las emociones de manera precisa han resultado controvertidos, la mayoría de los autores coincide en señalar que las emociones son fenómenos multifactoriales que ejercen una fuerte influencia sobre el comportamiento de las personas y en su adaptación al medio (Moltó Brotons, 1995; Moltó et al., 1999).

Wundt (1896) fue el primero en proponer un modelo dimensional al sugerir que la emoción es el resultado de variaciones en las dimensiones de placer y activación. Posteriormente, los análisis factoriales sobre el diferencial semántico (Osgood, Suci, \& Tannembaum, 1957), que evaluaba el lenguaje afectivo (Smith \& Ellsworth, 1985), y los resultados de los autoinformes verbales sobre distintos estados emocionales (Watson \& Tellegen, 1985) han llevado al desarrollo de una teoría acerca de la emoción en la que la valencia afectiva juega un papel fundamental.

La aproximación teórica mencionada acerca de las emociones fue retomada por Lang (1968, 1979); la misma integra resultados de las líneas anteriormente citadas sumadas a investigaciones en el ámbito del aprendizaje animal (Dickinson \& Dearing, 1979). El autor propone una visión bifásica de las emociones en la que estas son disposiciones para la acción que resultan de la activación de dos sistemas motivacionales: el apetitivo y el aversivo. Estos sistemas están integrados en circuitos neurales del cerebro y relacionados con estructuras cerebrales que regulan los sistemas somático y autónomo implicados en la atención y en la acción (Davis \& Lang, 2003; LeDoux, 2000). Según este modelo, se identifican dos dimensiones afectivas básicas: la valencia afectiva y el arousal o activación. La primera indicaría qué sistema motivacional se ha activado y la segunda reflejaría la intensidad de la misma (Cacioppo \& Berntson, 1994). El sistema motivacional apetitivo, se activa en contextos que promueven la supervivencia, con un repertorio conductual de búsqueda de alimento, sexo y prestación de cuidados. Por el contrario, el sistema de defensa, se activa principalmente en contextos relacionados con la amenaza, vinculados a comportamientos de lucha o huida (Bradley, Codispoti, Cuthbert, \& Lang, 2001; Lang, 1995; Reisberg \& Heuer, 2004).

La dimensión de dominancia se ha encontrado de manera menos consistente y con un peso explicativo menor; esto se refiere al grado de control percibido sobre la respuesta emocional, que correlaciona positivamente con la dimensión de valencia y, por tanto, añade poco valor discriminativo a las dos dimensiones afectivas básicas (Bradley et al., 2001; Bradley, Greenwald, \& Hamm, 1993; Diener, Smith, \& Fujita, 1995). Asimismo, la evaluación de la dominancia presenta dificultad en niños y adolescentes, dada la complejidad de la variable y el tipo de respuesta esperada; por lo cual en algunos estudios, se han considerado solamente las dos dimensiones básicas (Betella \& Verschure, 2016; Huang et al., 2015; Smith, McIver, Di Nella, \& Crease, 2011).

La principal fuente de evidencia a favor del modelo proviene de estudios dirigidos a conocer los circuitos neurales y fisiológicos de los sistemas motivacionales, utilizando como paradigma de investigación, la visualización de imágenes afectivas. En este sentido, el instrumento para el estudio experimental de las emociones diseñado por Lang, Bradley, y Cuthbert (2008), denominado International Affective Picture System (IAPS), es una colección de fotografías que representan objetos, personas, paisajes y situaciones de la vida cotidiana. Las imágenes han sido evaluadas por hombres y mujeres en las dimensiones afectivas de valencia (nivel de desagrado / agrado de la imagen) y arousal (nivel de calma / activación que provoca la imagen). Además, los autores han seleccionado y baremado un set de imágenes para su utilización en niños y adolescentes (McManis, Bradley, Berg, Cuthbert, \& Lang, 2001).

La ventaja de utilizar esta base baremada de estímulos emocionales es que permite la selección precisa de los estímulos en función de su posición en el espacio afectivo y su estandarización está basada en datos teóricos y experimentales, en los cuales las reacciones emocionales que provocan son similares a aquellas que inducen los estímulos reales (Bradley \& Lang, 2007). Además, la inclinación diferente de la distribución de las imágenes en función de la valencia afectiva esto es, la mayor inclinación hacia las imágenes desagradables que las agradables- sugiere mecanismos de activación diferentes para los sistemas 
apetitivo y aversivo. Cuando los estímulos ambientales no son ni fuertemente amenazantes ni fuertemente atractivos, la tendencia a la aproximación será mayor que la tendencia a la evitación, lo que explicaría las reacciones exploratorias y de orientación típica de la vida cotidiana (Bradley \& Lang, 2000a; Dickinson \& Dearing, 1979).

Por otro lado, los datos psicofisiológicos y conductuales indican que las imágenes del IAPS pueden producir cambios mensurables y fiables en los sistemas autonómico, muscular facial y esquelético-reflejo, así como en el comportamiento de las personas (Cuthbert, Bradley, \& Lang, 1996; Lang, 1995; Lang et al., 2008). Estas respuestas fisiológicas, verbales y conductuales pueden ser organizadas en torno a las dimensiones de valencia y activación, respaldando de forma empírica el modelo teórico y la organización bifásica de las emociones.

Uno de los aportes del presente estudio es que, si bien se han realizado adaptaciones transculturales del IAPS en adultos en países como España (Moltó et al., 1999; Vila et al., 2001; Moltó Brotons et al., 2013), Chile (Dufey, Fernandez, \& Mayol, 2011; Silva, 2011), Argentina (Irrazabal, Aranguren, Zaldua, \& Di Giuliano, 2015), Colombia (Gantiva Díaz, Guerra Muñoz, \& Vila Castellar, 2011) y Brasil (Larsen Ribeiro, Pompéia, \& Amodeo Bueno, 2005), las mismas no han sido realizadas en la población infantojuvenil. Por lo tanto, el objetivo de la presente investigación fue analizar el comportamiento psicométrico del conjunto de imágenes específico para niños y adolescentes del IAPS para obtener los valores normativos en población argentina y evaluar las diferencias en tres grupos etarios. Se espera que los resultados permitan su implementación en estudios de neurociencia afectiva en niños y adolescentes argentinos.

\section{Método}

La presente investigación se realizó a través de un estudio descriptivo-comparativo con un diseño ex post facto, transversal.

\section{Participantes}

La muestra y la conformación de los grupos, se definió según el Reporte Técnico A-8 (Lang et al., 2008), en el que se presenta el manual de instrucciones y los baremos del IAPS para población estadounidense. Se conformó una muestra intencional, ajustándose a los lineamientos del estudio original en niños (Lang et al., 2008). Así, participaron 141 niños y adolescentes escolarizados de entre 7 y 14 años, con una edad media de 11,16 años (DE: 2,16), pertenecientes a instituciones educativas de nivel primario y secundario de la ciudad de Mar del Plata, Argentina. La muestra estuvo dividida en tres franjas etarias: entre 7 y 9 años $(n=33)$, entre 10 y 12 años $(n=$ $55)$ y entre 13 y 14 años $(n=53)$. En todos los casos, los niños y adolescentes participantes firmaron el asentimiento informado y sus padres o tutores firmaron el consentimiento informado, asegurándoles un trato confidencial de los resultados.

El instrumento utilizado fue el conjunto de imágenes específico para niños y adolescentes IAPS (Lang et al., 2008) que cuenta con 60 imágenes digitalizadas a color de diversas categorías semánticas representadas por objetos, personas, paisajes y situaciones de la vida cotidiana.

Los juicios evaluativos de los niños y adolescentes se midieron utilizando la versión impresa del Self-Assessment Manikin (SAM) (Bradley \& Lang, 1994; Lang et al., 2008), un instrumento de medida pictórico, no verbal, de fácil y rápida administración. El SAM es una escala compuesta por cinco pictogramas donde se utilizan figuras humanoides graduadas en intensidad para representar los rangos de las dimensiones analizadas. El mismo constituye un instrumento útil y adecuado para su implementación transcultural, no requiriendo el uso del lenguaje.

\section{Procedimiento}

Las imágenes fueron evaluadas en distintas sesiones en grupos reducidos (entre 15 y 20 participantes), equilibrando la proporción entre varones y mujeres. Para la estandarización del conjunto de fotografías se utilizaron tres secuencias distintas de presentación de las imágenes con el fin de balancear la posición de cada imagen en su conjunto. Se utilizó un set de tres fotografías de práctica, diferentes a las 60 imágenes utilizadas en la evaluación, que proporcionan una gama aproximada de los tipos de contenidos que se presentarán.

Las sesiones de evaluación de las imágenes se realizaron de forma grupal a la misma hora del 
día (de 10:00 a 13:00 hrs.), con la misma distancia de los participantes a la pantalla (entre 6 y 9 $\mathrm{m})$ y con idénticas condiciones de iluminación y tamaño de proyección de las imágenes $(1,30 \mathrm{~m}$ por 1,70 m), siguiendo las indicaciones de Lang et al. (2008). Se utilizó Microsoft Office Powerpoint 15.0 (2013) para la presentación de los estímulos.

Al inicio de cada sesión experimental, se explicó a los participantes la finalidad del estudio de manera clara y comprensible. Para segundo y tercer año del nivel primario, se destinó una mayor cantidad de tiempo para la explicar la actividad. Esto con el fin de facilitar la comprensión de la misma.

Los participantes evaluaron cada imagen en las dos dimensiones marcando con una " $\mathrm{X}$ " sobre una de las cinco figuras o en cualquiera de los cuatro espacios entre figuras, resultando una puntuación entre 1 [desagrado y activación mínima], 5 [valencia y arousal neutro] y 9 [agrado y activación máxima] por cada dimensión. En el SAM, la dimensión de valencia afectiva está representada en un extremo por una figura sonriendo y en el otro con una figura seria con el ceño fruncido. El arousal, en cambio, está representado en un extremo con una figura excitada con los ojos abiertos y en el otro extremo con una figura relajada y con los ojos cerrados.

En cada ensayo se presentó: (a) una diapositiva de presentación con la leyenda "Prepárense para evaluar la siguiente diapositiva en la línea X", que permaneció proyectada durante cinco segundos; (b) una imagen afectiva, que se proyectó durante seis segundos; y (c) una diapositiva con la siguiente instrucción de evaluación: "Por favor, evalúa la imagen en las dos dimensiones", que permaneció durante 20 segundos con el fin de que los participantes contaran con tiempo suficiente para completar las dos escalas de evaluación del SAM (Irrazabal et al., 2015; Lang et al., 2008; Moltó Brotons et al., 2013).

\section{Análisis de datos}

Para el procesamiento de los datos se utilizó el paquete estadístico IBM SPSS versión 19.0, Statistical Package for Social Sciences (George \& Mallery, 2003). En primer lugar, para analizar la fiabilidad se calculó alfa de Cronbach. Luego, se obtuvieron los estadísticos descriptivos de las 60 imágenes (media y desviación estándar) para el total de la muestra y para cada franja etaria. Asimismo, se realizaron análisis correlacionales mediante la obtención del coeficiente de correlación de Pearson para evaluar tanto el grado de relación entre las medias de valencia y arousal -para el total de la muestra y para cada franja etaria-, como la relación entre valencia agradable y arousal, y valencia desagradable y arousal.

Para analizar las diferencias de medias entre los tres grupos etarios respecto a la variable de activación para las imágenes del polo desagradable y las imágenes del polo agradable, se realizó un Anova. Como prueba post hoc se utilizó a Games-Howell, ya que la prueba de Levene indicó diferencias significativas, por lo que no hay varianzas iguales.

\section{Resultados}

\section{Fiabilidad del IAPS}

Los coeficientes de alfa de Cronbach para el conjunto de 60 imágenes del IAPS para niños y adolescentes han sido buenos para valencia $(, 84)$ y excelente para arousal $(94)$ de acuerdo con los criterios de George y Mallery (2003).

En el anexo se presentan las medias (M) y desviaciones estándar (DE) de las evaluaciones en las dimensiones de valencia y arousal de cada una de las 60 imágenes que conforman el conjunto de imágenes específico para niños y adolescentes del IAPS (Lang et al., 2008).

\section{El espacio bidimensional afectivo}

En la figura 1 se muestra la representación gráfica de las 60 imágenes, en el espacio bidimensional afectivo, definido por valencia y arousal, según las evaluaciones obtenidas por el total de los participantes. El eje vertical refleja la posición de cada imagen en la dimensión de valencia $(1$ = altamente desagradable, $5=$ neutra, $9=$ altamente agradable), mientras que el eje horizontal refleja su posición en la dimensión de arousal $(1=$ calma, $5=$ moderadamente activadora, 9 = altamente activadora). Cada punto de la gráfica representa la media de las evaluaciones del total de participantes para cada una de las imágenes. Cada uno de ellos está representado por un código de cuatro dígitos correspondientes al código internacional de imagen del IAPS. 


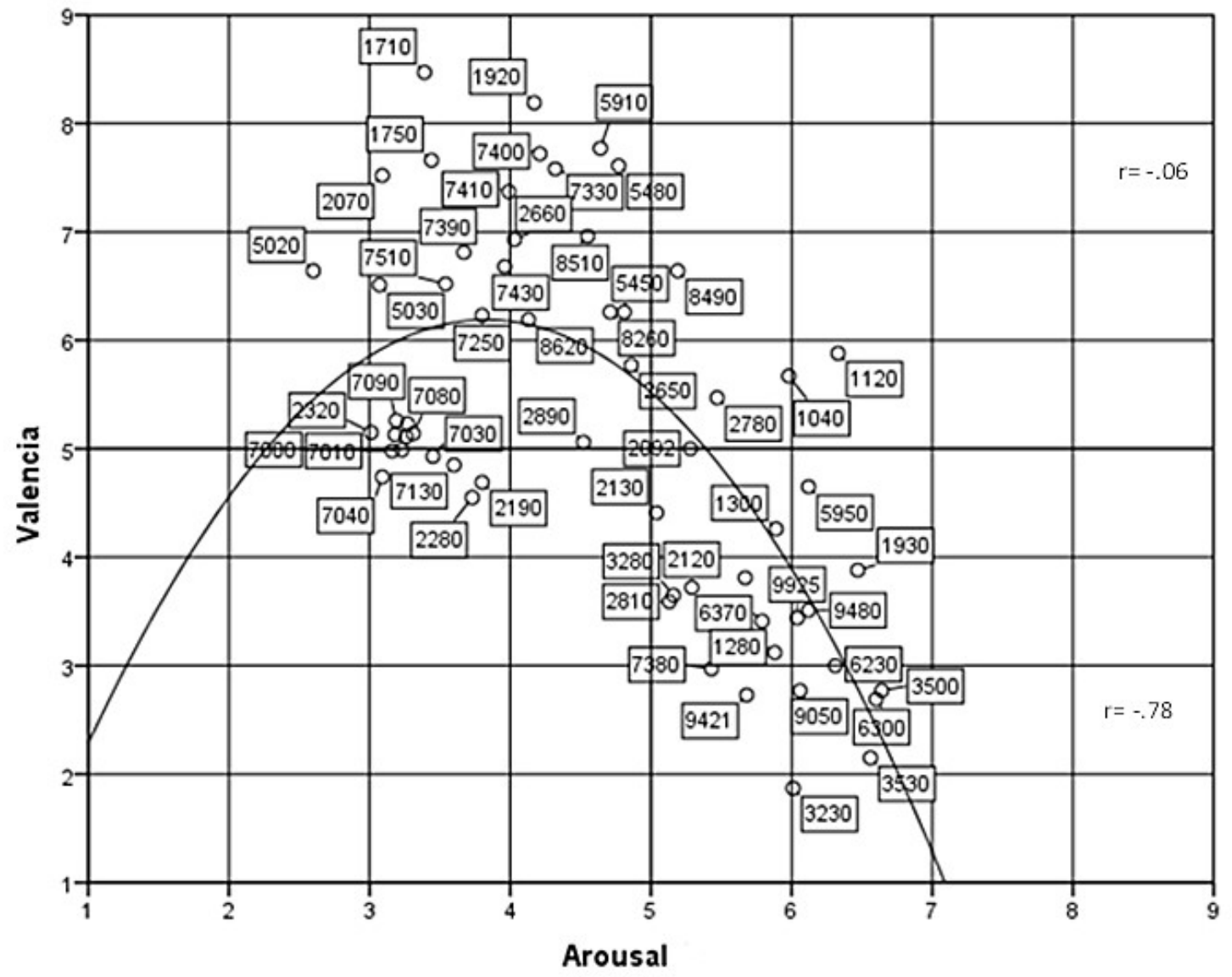

Figura 1. Distribución de las 60 imágenes del IAPS según las medias para el total de la muestra evaluada en las dimensiones de valencia y arousal.

En la figura 1, la distribución de las imágenes en el espacio afectivo muestra una correlación moderada negativa significativa entre la valencia y el arousal, a valores más altos en la primera se presentan valores más bajos en la segunda $(r(141)$ $=-, 60 ; p<, 05)$. Esta observación se vio confirmada estadísticamente por la relación cuadrática entre las evaluaciones de valencia afectiva y arousal $\left(r^{2}=, 46 ; p<, 05\right)$, que explicaría el $46 \%$ de la varianza.

Las imágenes consideradas agradables, es decir, las de los cuadrantes superiores, se distribuyen ocupando en su mayoría el cuadrante de baja activación, con una correlación débil y no significativa entre ambas variables $(r(141)=-, 06$; $p=$,74). Mientras que las imágenes desagradables, es decir las de los cuadrantes inferiores, se agrupan mayoritariamente en el cuadrante de alta activación con una correlación fuerte, negativa y significativa $(r(141)=-, 78 ; p<$ ,05), es decir, cuanto más desagradables, más activadoras resultan. De este modo, se muestra una mayor dispersión y menor inclinación de la activación para las imágenes agradables respecto de las desagradables. Asimismo, se observa un agrupamiento de imágenes con valencia neutra y muy baja activación.

Las imágenes con una base afectiva agradable y de baja activación corresponden a contenidos de cachorros $(1710)^{1}, \quad$ animales pequeños

\footnotetext{
${ }^{1}$ Los números entre paréntesis representan los códigos internacionales de las imágenes del IAPS.
} 
(1710/1750), flores (5020/5030), bebés (2070/2660), helado (2650/7330/7390), y golosinas $(7400 / 7410 / 7430)$.

Por otro lado, las imágenes que resultaron más desagradables son aquellas vinculadas a catástrofes e incidentes (9050/9925), arma de fuego (6230), soldados heridos (9421), hombre hospitalizado (3230), y huesos y cráneos humanos
(9480); y las imágenes desagradables con valores más altos en arousal representan escenas violentas con armas de fuego y arma blanca (3500/3530/6300), y animales agresivos (1930).

En las figuras 2, 3 y 4 se representa la distribución de las imágenes en el espacio bidimensional afectivo para las tres franjas etarias.

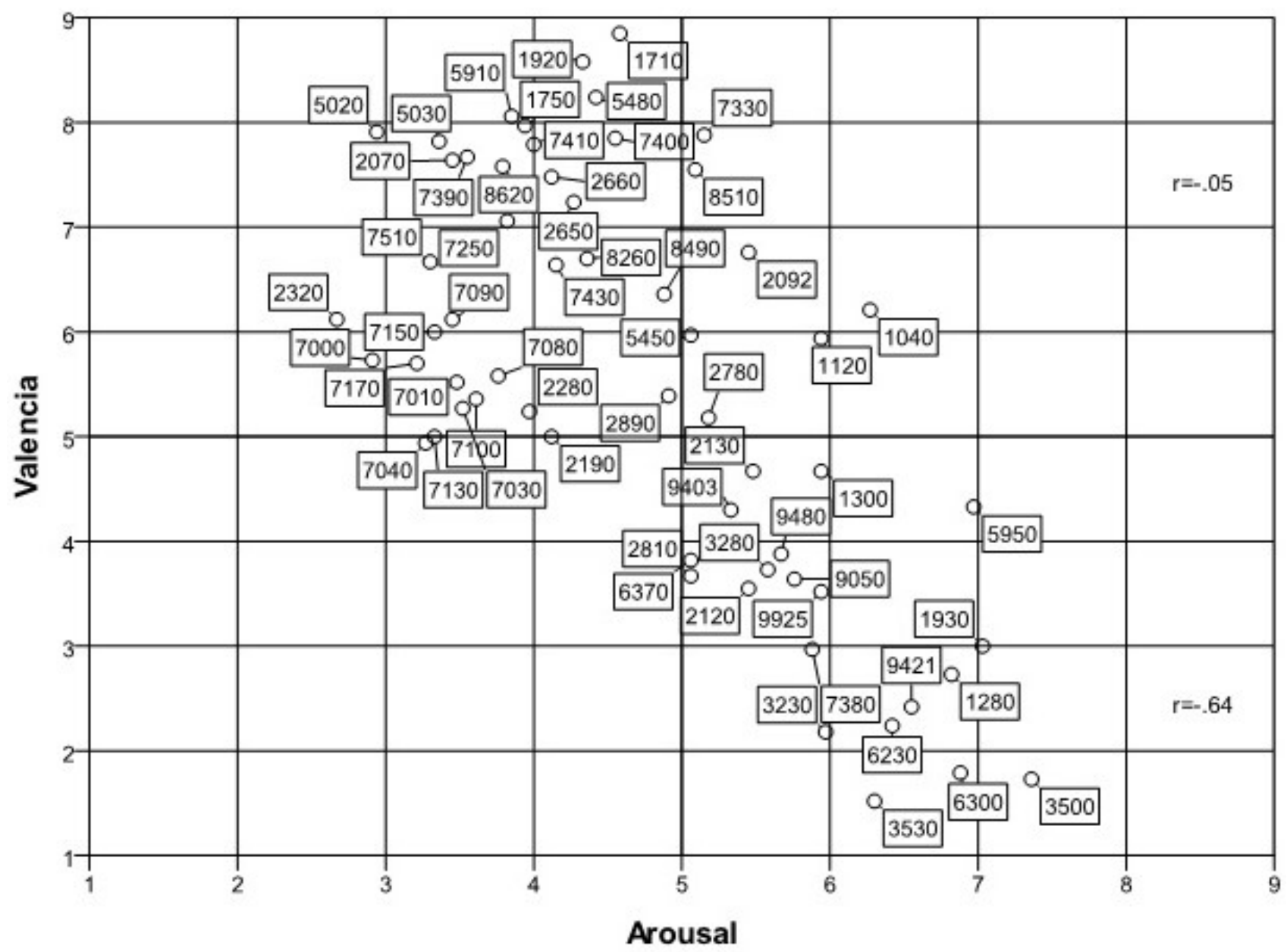

Figura 2. Distribución de las 60 imágenes del IAPS según las medias para los niños de 7 a 9 años en las dimensiones de valencia y arousal. 


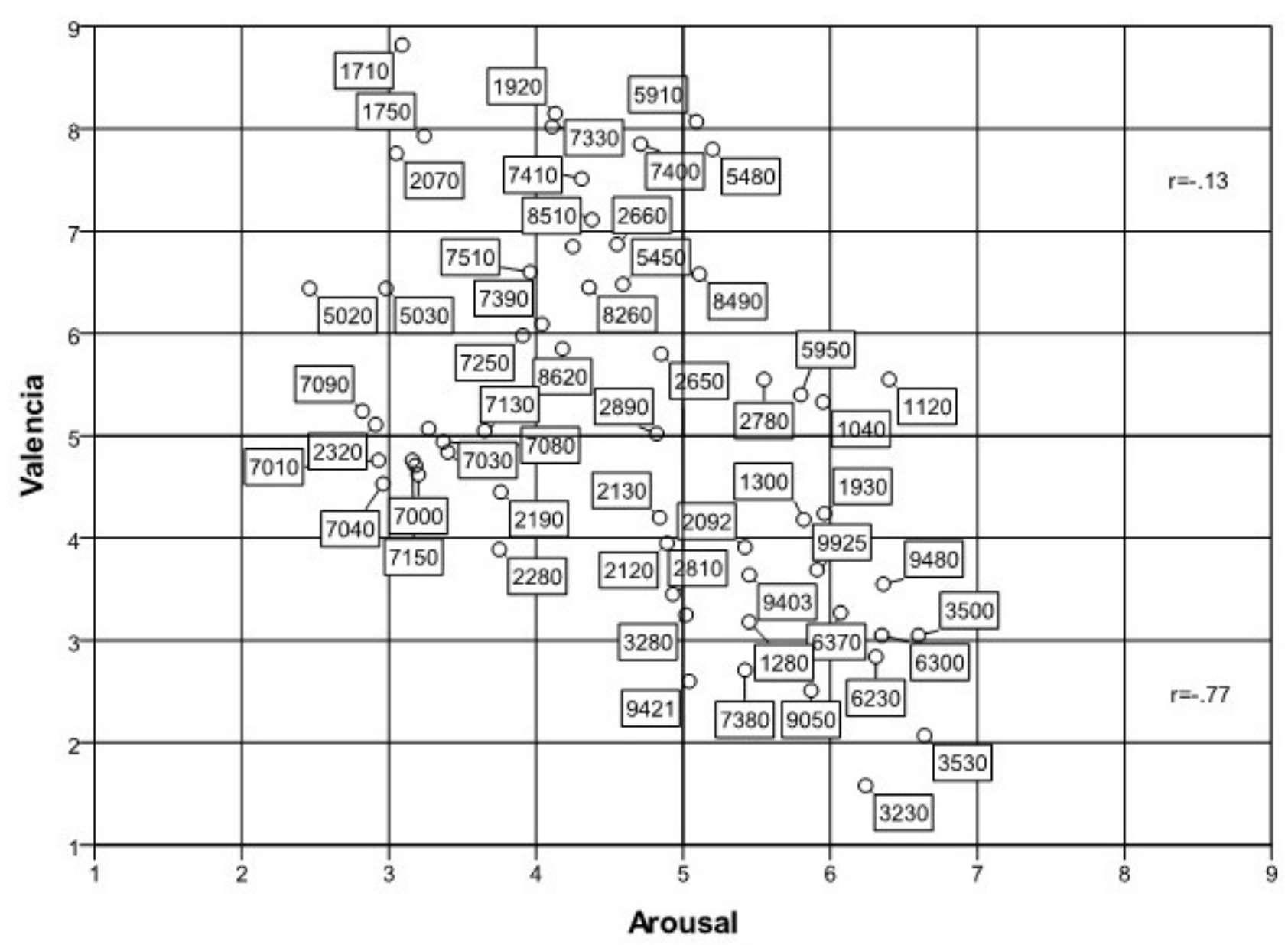

Figura 3. Distribución de las 60 imágenes del IAPS según las medias para los niños de 10 a 12 años en las dimensiones de valencia y arousal. 


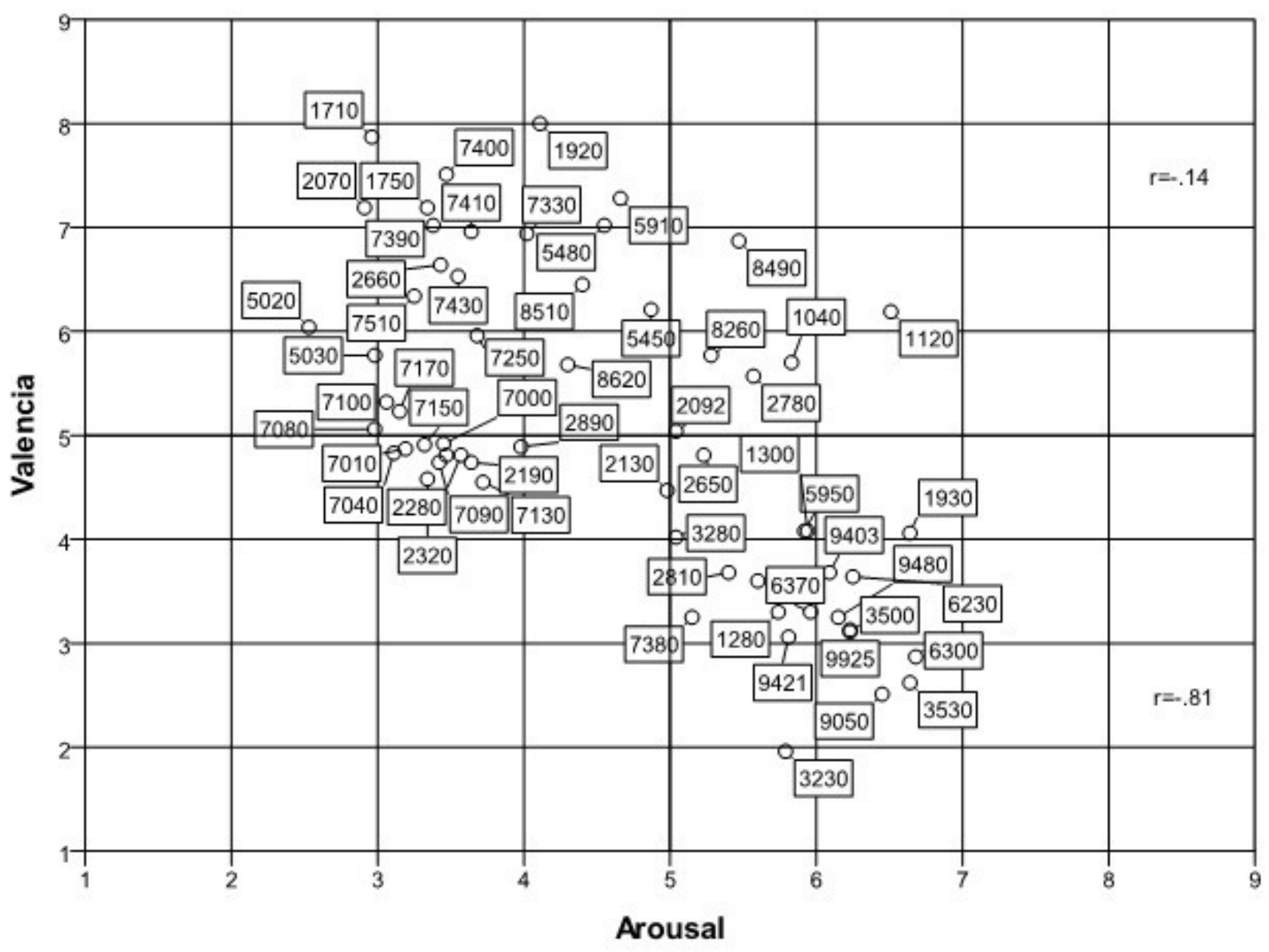

Figura 4. Distribución de las 60 imágenes del IAPS según las medias para los niños de 13-14 años $(n=53)$ en las dimensiones de valencia y arousal.

Se puede observar en todas las figuras una distribución similar a la del total de la muestra, agrupándose la mayor parte de las imágenes en el cuadrante activador-desagradable $y$ en el cuadrante calmo-agradable. Se observa mayor inclinación y menor dispersión en la distribución del cuadrante inferior activador-desagradable: grupo entre 7 y 9 años: $r(33)=-, 64 ; p<, 000$; grupo entre 10 y 12 años: $r(55)=-, 77 ; p<, 000 \mathrm{y}$ grupo entre 13 y 14 años: $r(53)=-, 81 ; p<, 000$. Hay una menor inclinación y mayor dispersión en la distribución del cuadrante superior calmo- agradable: grupo entre 7 y 9 años: $r(33)=, 05 ; p=$ ,72; grupo entre 10 y 12 años: $r(55)=-, 13 ; p=$ , 47 y grupo entre 13y 14 años: $r(53)=-, 14 ; p=$ ,45. Y en los adolescentes de entre 13 y 14 años $(r(53)=-, 81 ; p<, 000)$, se observa una menor dispersión en las imágenes desagradables en comparación con los otros dos grupos: 7 y 9 años $(r(33)=-, 64 ; p<, 000)$; y 10 y 12 años $(r(55)=$ ,77; $p<, 000)$.

En la tabla 2 se presentan los estadísticos descriptivos y prueba $t$ para las dimensiones de valencia y arousal según sexo. 
Tabla 2

Estadisticos descriptivos y prueba $t$

\begin{tabular}{cccc}
\cline { 2 - 4 } & Mujeres & Varones & \\
& $M(D E)$ & $M(D E)$ & Sig. \\
\hline Valencia & $5,18(1,80)$ & $5,23(1,61)$ &, 87 \\
Arousal & $4,53(1,08)$ & $4,71(1,30)$ &, 41 \\
\hline
\end{tabular}

Respecto al análisis por género, las mujeres han puntuado más bajo, tanto en valencia como en arousal, en comparación con los varones, sin diferencias estadísticamente significativas.

En la tabla 3 se presentan los resultados de las diferencias de medias (Anova) entre los tres grupos etarios respecto a la variable de activación para las imágenes del polo desagradable (puntuación de 1 a 5) y las imágenes del polo agradable (puntuación de 5 a 9).

En la tabla 3 puede observarse que la cantidad de imágenes de cada polo para los diferentes grupos etarios son semejantes. Las imágenes del polo desagradable presentan mayor arousal respecto a las imágenes del polo agradable.
Respecto a la comparación de medias de activación para cada polo en función de la edad, se puede observar que, en el polo desagradable, las imágenes presentan mayor activación en los niños de 7 a 9 años y menor activación en el grupo de niños de 10 a 12 años, con diferencias significativas entre los grupos de 7 a 9 y de 10 a 12 y los grupos de 7 a 9 y de 13 y 14 años. Y respecto a la comparación de medias para el polo agradable los datos indican que las imágenes presentan mayor activación en el grupo de niños de 10 a 12 años y menor activación en el grupo de adolescentes de 13 y 14 años, no observándose diferencias significativas entre los grupos etarios.

Tabla 3

Diferencia de medias en la dimensión de arousal

\begin{tabular}{|c|c|c|c|c|c|c|c|c|c|c|c|c|}
\hline \multirow[b]{2}{*}{ Valencia } & \multicolumn{3}{|c|}{7 a 9 años } & \multicolumn{3}{|c|}{10 a 12 años } & \multicolumn{3}{|c|}{13 y 14 años } & \multicolumn{3}{|c|}{ Comparaciones } \\
\hline & $N$ & $M$ & $D E$ & $N$ & $M$ & $D E$ & $N$ & $M$ & $D E$ & $\begin{array}{c}7 \text { a } 9- \\
10 \text { a } 12\end{array}$ & $\begin{array}{c}7 \text { a } 9- \\
13 \text { y } 14\end{array}$ & $\begin{array}{l}10 \text { a } 12 \\
13 \text { y } 14\end{array}$ \\
\hline Polo desagradable & 21 & 5,93 &, 90 & 29 & 4,97 & 1,25 & 32 & 5,06 & 1,25 &, $00 *$ &, $01 *$ &, 95 \\
\hline Polo agradable & 39 & 4,1 & 83 & 31 & 4,24 &, 98 & 28 & 4,03 & 1,04 &, 79 &, 95 &, 69 \\
\hline
\end{tabular}

Nota $^{*} p<, 05 . N=$ número de imágenes; $M=$ media; $D E=$ desviación estándar.

\section{Discusión y conclusiones}

Las respuestas a estímulos afectivos en adultos han demostrado un patrón constante (Lang et. al., 2008; Moltó et al., 1999; Moltó Brotons et al., 2013; Vila Castellar et al., 2001). Sin embargo, pocas investigaciones dan cuenta del procesamiento emocional en niños y adolescentes (Lang, Greenwald, Bradley, \& Hamm, 1993; McManis et al., 2001), estando la mayor parte de las mis- mas enfocada a población clínica (Mathews \& MacLeod, 1994; Sharp, Petersen, \& Goodyer, 2008; Sharp, Van Goozen, \& Goodyer, 2006). Por tanto, el objetivo de este estudio fue analizar el comportamiento psicométrico del conjunto de imágenes específico para niños y adolescentes del International Affective Picture System (IAPS) para obtener los valores normativos en población argentina y evaluar las diferencias en tres grupos etarios. 
La distribución de las imágenes en el espacio afectivo evidenció una relación inversa entre valencia y arousal, dando cuenta de que las imágenes con menor agradabilidad resultan más activadoras, en respuesta a mecanismos de activación diferentes de los sistemas apetitivo y aversivo tal como lo plantea la teoría bioinformacional de Lang et al. (2008). La ausencia de imágenes en el área poco activadora y desagradable del espacio bidimensional es coincidente con los datos de McManis et al. (2001) en niños y adolescentes de Estados Unidos.

Asimismo, la distribución de las imágenes agradables presentó una mayor dispersión y menor inclinación de la activación respecto de las imágenes desagradables en consonancia con los resultados presentados por McManis et al. (2001) en población infantil, y por Lang et al. (2008) y Moltó Brotons et al. (2013) en población adulta.

En concordancia con el modelo se observa una alta concentración de imágenes en el área neutracalma que se proyectan fuertemente hacia el polo desagradable activador, con una concentración muy baja en el polo agradable activador.

Gran parte de las adaptaciones transculturales del IAPS en adultos y niños han reafirmado el patrón en forma de boomerang del espacio bidimensional afectivo con altos niveles de activación para valores extremos de valencia. Sin embargo, adaptaciones realizadas en Brasil (Larsen Ribeiro et al., 2005) y Alemania (Grühn \& Scheibe, 2008) con población adulta han obtenido puntuaciones más lineales, atribuyendo menos activación a imágenes agradables y más activación a imágenes neutras y desagradables. En el presente estudio se observó la ausencia de imágenes en el área muy activadora y agradable que podría deberse, al igual que las adaptaciones citadas, a variaciones culturales, siendo este modulador de la personalidad y fuente de experiencias diferentes (Kuppens, Tuerlinckx, Russell, \& Barrett, 2013). Tal como plantea Aluja et al. (2015) la influencia cultural afecta la evaluación subjetiva de algunos contenidos de las imágenes del IAPS, más allá de la validez transcultural que tenga el instrumento de manera global. Lang y Davis (2006) le adjudican a la valencia un origen neurobiológico resultado de un proceso de adaptación y evolución humana, a diferencia del arousal que estaría más influenciada por factores socioculturales, de habituación y de aprendizaje (Cuenya, Fosacheca, Mustaca, \&
Kamenetzky, 2011; Cuenya, Kamenetzky, Fosacheca, \& Mustaca, 2013).

En cambio, la mayor concentración de imágenes en el cuadrante activador desagradable, presente tanto en niños y adolescentes de nuestro estudio como en el estudio de Irrazabal et al. (2015) en adultos argentinos, indicaría una fuerte inclinación hacia el sistema aversivo cuando la activación es alta. Esto se traduce en una mayor cantidad de energía invertida ante situaciones peligrosas, tendiente a la lucha o huida, demostrando un sesgo hacia la negatividad, con claras funciones adaptativas y de supervivencia (Miller, 1959). Esto último da cuenta de una mayor tendencia a responder con reacciones de evitación o escape, que con reacciones de acercamiento cuando aumenta el nivel de activación ante estímulos de valencia desagradable (Bradley \& Lang, 2000b; Bradley et al., 2001; Lang, 1995; Lang, Davis, \& Öhman, 2000).

Diversos estudios dieron cuenta de que la amígdala participa en la codificación de estímulos de alta activación, explicando cómo a través de la valencia negativa se puede comprender los efectos de la activación en la red de memoria emocional (Kensinger \& Schacter, 2006; Kensinger, 2007; Mickley Steinmetz, Addis, \& Kensinger, 2010). Existe una vía a través de la cual la amígdala influye en la cantidad de la entrada sensorial que es recibida por el hipocampo, lo que explica por qué acontecimientos negativos son a veces recordados vívidamente, y con detalle sensorial, incluso cuando no son elevados en activación (Kensinger \& Corkin, 2003; Lang, 2010). A su vez en la emoción se activan redes complejas de información almacenadas en la memoria, que pueden generar diferentes tipos de respuestas, e incluso procesarse sin ninguna acción externa (Foa, Ilai, McCarthy, Shoyer, \& Murdock, 1993; Redondo \& Fernández-Rey, 2010; Reisberg \& Heuer, 2004).

En cuanto a las limitaciones del presente estudio, se considera conveniente ampliar la muestra poblacional a fin de permitir una mayor generalización de los resultados. El IAPS es un instrumento ampliamente utilizado en investigación experimental, sin embargo, podría presentar algunas restricciones. En primera instancia, la base de imágenes no se distribuye en categorías semánticas explícitas y la calidad de las fotografías no están acordes con los parámetros actuales de cali- 
dad visual (Weinberg \& Hajcak, 2010). El formato de las imágenes no es homogéneo es decir, varía en resolución, tamaño, forma, luminosidad, variables que influyen en el procesamiento afectivo de los estímulos visuales (Bradley, Hamby, Löw, \& Lang, 2007; Marchewka, Zurawski, Jednoróg, \& Grabowska, 2014; Nordström \& Wiens, 2012). Nuevas bases de datos como el Nencki Affective Picture System (Marchewka et al., 2014) han sumado a los valores de valencia y arousal, propiedades físicas de las imágenes para una clasificación más específica del estímulo. La variable dominancia dentro de este último estudio también ha sido excluida de su estudio en adultos por su poco valor discriminativo con respecto a la valencia.

A partir de la discusión que antecede surge como futura línea de trabajo la comparación de los resultados de este estudio con los baremos realizados en Estados Unidos por Lang et al. (2008). Asimismo resulta de interés el análisis y comparación de distintos mecanismos motivacionales en niños y adolescentes que permitan relacionar indicadores fisiológicos, psicológicos y comportamentales. Estos estudios permitirían diferenciar según sexo los patrones de respuesta específicos para los sistemas aversivo y apetitivo.

Además, se requieren de estudios para determinar la fiabilidad de las respuestas evaluativas de los niños y adolescentes para estímulos afectivos. Nuevas investigaciones en IAPS con niños, adolescentes y adultos permitirán analizar el desarrollo del procesamiento emocional. Variables tales como la dominancia, el interés, el grado de expectativa y la influencia relativa de una imagen sobre otra, son algunos de los aspectos a profundizar en el campo de la neurociencia afectiva.

A partir de las evidencias presentadas se concluye que el presente estudio permite brindar aportes empíricos sobre el comportamiento psicométrico del IAPS en población argentina infanto-juvenil, el cual podría ser utilizado en tareas experimentales y en el desarrollo de estudios acerca de las emociones en niños y adolescentes, y su relación con variables cognitivas. Debido a la escasa producción científica en el IAPS para esta población, se considera un aporte para su implementación en los ámbitos investigativo educativo y clínico.

\section{Referencias}

Aluja, A., Rossier, J., Blanch, A., Blanco, E., MartíGuiu, M., \& Balada, F. (2015). Personality effects and sex differences on the International Affective Picture System (IAPS): A Spanish and Swiss study. Personality and Individual Differences, 77(1), 143-148. https://doi.org/10.1016/j.paid.2014.12.058

Betella, A. \& Verschure, P. F. M. J. (2016). The affective slider: A Digital Self-Assessment Scale for the measurement of human emotions. PLoS ONE 11(2), 1-11. https://doi.org/10.1371/journal.pone.0148037

Bradley, M. M. \& Lang, P. J. (1994). Measuring emotion: The self-assessment manikin and the semantic differential. Journal of Behavior Therapy and Experimental Psychiatry, 25(1), 49-59. https://doi.org/10.1016/0005-7916(94)90063-9

Bradley, M. M. \& Lang, P. J. (2000a). Emotion and motivation. En J. Cacioppo, L. G. Tassinary, \& G. G. Berntson (Eds.), Handbook of psychophysiology (pp. 602-642). New York, New York: Cambridge University Press.

Bradley, M. M. \& Lang, P. J. (2000b). Measuring emotion: Behavior, feeling and physiology. En R. Lane \& L. Nadel (Eds.), Cognitive neuroscience of emotion. (pp. 242- 276). New York, New York: Oxford University Press.

Bradley, M. M. \& Lang, P. J. (2007). The International Affective Picture System (IAPS) in the study of emotion and attention. En J. A. Coan \& J. J. B. Allen (Eds.), Handbook of emotion elicitation and assessment (pp. 29-46). New York, New York: Cambridge University Press.

Bradley, M. M., Codispoti, M., Cuthbert, B. N., \& Lang, P. J. (2001). Emotion and motivation I: Defensive and appetitive reactions in picture processing. Emotion, 1(3), 276-298. http://dx.doi.org/10.1037/1528-3542.1.3.276

Bradley, M. M., Greenwald, M. K., \& Hamm, A. O. (1993). Affective picture processing. En N. Birbaumer \& A. Öhman (Eds). The structure of emotion. Psychophysiological, cognitive and clinical aspects (pp. 48-65). Seattle, Washington: Hogrefe and Huber.

Bradley, M. M., Hamby, S., Löw, A., \& Lang, P. J. (2007). Brain potentials in perception: Picture complexity and emotional arousal. Psychophysiology, 44(3), 364-373. http://doi.org/frn $48 \mathrm{j}$

Cacioppo, J. T. \& Berntson, G. G. (1994). Relationships between attitudes and evaluative space: A critical review with emphasis on the separability of 
positive and negative substrates. Psychological Bulletin, 115(3), 401-423.

http://dx.doi.org/10.1037/0033-2909.115.3.401

Cuenya, L., Fosacheca, S., Mustaca, A., \& Kamenetzky, G. (2011). Efectos del aislamiento en la adultez sobre el dolor y la frustración. Psicológica: Revista de Metodología y Psicología Experimental, 32(1), 49-63.

Recuperado de https://goo.gl/dBKH52

Cuenya, L., Kamenetzky, G., Fosacheca, S., \& Mustaca, A. E. (2013). Successive negative contrast effect on the emotional evaluation of images. Annals of Psychology, 29(3), 944-952. http://dx.doi.org/10.6018/analesps.29.3.150861

Cuthbert, B. N., Bradley, M. M., \& Lang, P. J. (1996). Probing picture perception: Activation and emotion, Psychophysiology, 33(1), 103-111. http://doi.org/c58qn5

Davis, M. \& Lang, P. J. (2003). Emotion. En M. Gallagher, R. J. Nelson, \& Weiner, I. B. (Eds.), Handbook of psychology volume 3, biological psychology (pp. 405-439). New York, New York: John Wiley \& Sons, Inc.

Dickinson, A. \& Dearing, M. F. (1979). Appetitiveaversive interactions and inhibitory processes. En A. Dickinson \& R. A. Boakes (Eds.), Mechanisms of learning and motivation (pp. 203-231). Hillsdale, New Jersey: Lawrence Erlbaum.

Diener, E., Smith, H., \& Fujita, F. (1995). The personality structure of affect. Journal of Personality and Social Psychology, 69(1), 130-141.

Recuperado de https://goo.gl/FKbnZM

Dufey, M., Fernández, A. M., \& Mayol, R. (2011). Adding support to cross-cultural emotional assessment: Validation of the International Affective Picture System in a Chilean sample. Universitas Psychologica, 10(2), 521-533.

Recuperado de https://goo.gl/bbnSJj

Foa, E. B., Ilai, D., McCarthy, P. R., Shoyer, B., \& Murdock, T. (1993). Information processing in obsessive-compulsive disorder. Cognitive Therapy and Research, 17(2), 173-189.

Recuperado de https://goo.gl/sH7vVS

Gantiva Díaz, C. A., Guerra Muñoz, P., \& Vila Castellar, J. (2011). Validación colombiana del sistema internacional de imágenes afectivas: evidencias del origen transcultural de la emoción. Acta Colombiana de Psicología, 14(2), 103-111.

Recuperado de https://goo.gl/4Z2b73

George, D. \& Mallery, P. (2003). SPSS for Windows step by step: A simple guide and reference fourth edition (11.0 update). Boston, Massachusetts: Allyn \& Bacon.

Grühn, D. \& Scheibe, S. (2008). Age-related differences in valence and arousal ratings of pictures from the International Affective Picture System
(IAPS): Do ratings become more extreme with age? Behavior Research Methods, 40(2), 512-521. Recuperado de https://goo.gl/g2Mt6W

Huang, J., Xu, D., Peterson, B. S., Hu, J., Cao, L., Wei, N., ... Hu, S. (2015). Affective reactions differ between Chinese and American healthy young adults: A cross-cultural study using the international affective picture system. BMC psychiatry, 15(60), 2-7.

http://dx.doi.org/10.1186/s12888-015-0442-9

Irrazabal, N., Aranguren, M., Zaldua, E., \& Di Giuliano, N. (2015). Datos normativos del Sistema Internacional de Imágenes Afectivas (IAPS) en una muestra argentina. Revista Argentina de Ciencias del Comportamiento, 7(3), 34-50.

Recuperado de https://goo.gl/fUCxCp

Kensinger, E. A. (2007). Negative emotion enhances memory accuracy behavioral and neuroimaging evidence. Current Directions in Psychological Science, 16(4), 213-218.

http://doi.org/dgzzhc

Kensinger, E. A. \& Corkin, S. (2003). Memory enhancement for emotional words: Are emotional words more vividly remembered than neutral words? Memory \& Cognition, 31(8), 1169-1180. http://dx.doi.org/10.3758/BF03195800

Kensinger, E. A. \& Schacter, D. L. (2006). Amygdala activity is associated with the successful encoding of item, but not source, information for positive and negative stimuli. The Journal of Neuroscience, 26(9), 2564-2570.

http://doi.org/dckrrd

Kuppens, P., Tuerlinckx, F., Russell, J. A., \& Barrett, L. F. (2013). The relation between valence and arousal in subjective experience. Psychological Bulletin, 139(4), 917-940. http://dx.doi.org/10.1037/a0030811

Lang, P. J. (1968). Fear reduction and fear behavior problems in treating a construct. En J. M. Schlien (Ed.), Research in psychotherapy volume III (pp. 90-102). http://dx.doi.org/10.1037/10546-004

Lang, P. J. (1979). A bio-infomational theory of emotional imagery. Psychophysiology, 16(6), 495-512. http://doi.org/d3j9zb

Lang, P. J. (1995). The emotion probe: Studies of motivation and attention. American Psychologist, 50(5), 372-385. http://dx.doi.org/10.1037//0003-066X.50.5.372

Lang, P. J. (2010). Emotion and motivation: Toward consensus definitions and a common research purpose. Emotion Review, 2(3), 229-233. http://dx.doi.org/10.1177/1754073910361984

Lang, P. J., Bradley, M. M., \& Cuthbert, B. N. (2008). International Affective Picture System (IAPS): Affective ratings of pictures and instruction manual. 
Technical Report A-8. Gainesville, Florida: The Center for Research in Psychophysiology, University of Florida.

Lang, P. J., Davis, M., \& Öhman, A. (2000). Fear and anxiety: Animal models and human cognitive psychophysiology. Journal of Affective Disorders, 61(3), 137-159. http://dx.doi.org/10.1016/S0165-0327(00)00343-8

Lang, P. J., Greenwald, M. K., Bradley, M. M., \& Hamm, A. O. (1993). Looking at pictures: Affective, facial, visceral, and behavioral reactions. Psychophysiology, 30(3), 261-273.

http://doi.org/fwqnsw

Larsen Ribeiro, R., Pompéia, S., \& Amodeo Bueno, O. F. (2005). Comparison of Brazilian and American norms for the International Affective Picture System (IAPS). Revista Brasilera de Psiquiatría. 27(3), 208-215. http://doi.org/b7fxb8

LeDoux, J. (2000). Cognitive-emotional interactions: Listen to the brain. En R. D. Lane \& L. Nadel (Eds.), Cognitive neuroscience of emotion (pp. 129-155). Nueva York, New York: Oxford University Press.

Marchewka, A., Zurawski, L., Jednoróg, K., \& Grabowska, A. (2014). The Nencki Affective Picture System (NAPS): Introduction to a novel, standardized, wide-range, high-quality, realistic picture database. Behavior Research Methods, 46(2), 596-610.

http://dx.doi.org/0.3758/s13428-013-0379-1

Mathews, A. \& MacLeod, C. (1994). Cognitive approaches to emotion and emotional disorders. Annual Review of Psychology, 45(1), 25-50. $\mathrm{http}: / /$ doi.org/dsns $3 \mathrm{t}$

McManis, M. H., Bradley, M. M., Berg, W. K., Cuthberh, B. N., \& Lang, P. J. (2001). Emotional reaction in children: Verbal, physiological and behavioral responses of affective pictures. Psychophysiology, 38(2), 222-231.

http://dx.doi.org/10.1111/1469-8986.3820222

Mickley Steinmetz, K. R., Addis, D. R., \& Kensinger, E. A. (2010). The effect of arousal on the emotional memory network depends on valence. Neuroimage, 53(1), 318-324. http://doi.org/dw3pk5

Miller, N. E. (1959). Liberalization of basic S-R concepts: Extensions to conflict behavior, motivation, and social learning. En S. Koch (Ed.), Psychology: A study of a science (pp. 196-292). New York, New York: McGraw-Hill.

Moltó Brotons, J. (1995). Psicología de las emociones. Entre la biología y la cultura. Valencia, España: Albatros ediciones.

Moltó, J., Montañés, S., Poy, R., Segarra, P., Pastor, M. C., Tormo, M. P., ... Vila, J. (1999). Un nuevo método para el estudio experimental de las emociones: el International Affective Picture System \| (IAPS). Adaptación española. Revista de Psicologia General y Aplicada, 52(1), 55-87.

Recuperado de https://goo.gl/BgrtwH

Moltó Broyons, J., Segarra, P., Lopez Penadés, R., Esteller Á., Fonfría A., Pastor Verchili, M. C. \& Poy Gil, R. (2013). Adaptación española del International Affective Picture System (IAPS). Tercera parte. Anales de Psicología, 29(3), 965-984. http://dx.doi.org/10.6018/analesps.29.3.153591

Nordström, H. \& Wiens, S. (2012). Emotional eventrelated potentials are larger to figures than scenes but are similarly reduced by inattention. $\mathrm{BMCNeu-}$ roscience, 13(49), 1-10.

http://dx.doi.org/10.1186/1471-2202-13-49

Osgood, C., Suci, G., \& Tannembaum, P. (1957). The measurement of meaning. Urbana, Illinois: University of Illinois.

Redondo, J. \& Fernández-Rey, J. (2010). Reconocimiento de fotografías de contenido emocional: efectos de la valencia cuando se controla el arousal. Psicológica 31(1), 65-86.

Recuperado de https://goo.gl/KaQ4fR

Reisberg, D. \& Heuer, F. (2004). Memory for emotional events. En D. Reisberg \& P. Hertel (Eds.). Memory and emotion (pp. 3-41). Nueva York, New York: Oxford University Press.

Sharp, C., Petersen, N., \& Goodyer, I. (2008). Emotional reactivity and the emergence of conduct problems and emotional symptoms in 7-to 11-yearolds: A 1-year follow-up study. Journal of the American Academy of Child \& Adolescent Psychiatry, 47(5), 565-573.

http://dx.doi.org/10.1097/CHI.0b013e31816765d4

Sharp, C., Van Goozen, S., \& Goodyer I. (2006). Children's subjective emotional reactivity to affective pictures: Gender differences and their antisocial correlates in an unselected sample of 7-11-yearolds. Journal of Child Psychology and Psychiatry 47(2), 143-150.

http://doi.org/fd7rj5

Silva, R. J. (2011). International Affective Picture System (IAPS) in Chile: A cross-cultural adaptation and validation study. Terapia Psicológica, 29(2), 251-258.

http://doi.org/fzw3q4

Smith, C. A. \& Ellsworth, P. C. (1985). Patterns of cognitive appraisal in emotion. Journal of Personality and Social Psychology, 48(4), 813-838. http://dx.doi.org/10.1037/0022-3514.48.4.813

Smith, S. D., McIver, T. A., Di Nella, M. S., \& Crease, M. L. (2011). The effects of valence and arousal on the emotional modulation of time perception: Evidence for multiple stages of processing. Emotion, 11(6), 1305-1313. 
http://dx.doi.org/10.1037/a0026145

Vila Castellar, J., Blasa Sánchez, M., Ramírez Uclés, I., Fernández Santaella, M. C., Cobos Alvares, M. P., Rodríguez Fernández, S., ... Moltó, J. (2001). El Sistema Internacional de Imágenes Afectivas (IAPS): adaptación española. Segunda parte. Revista de Psicología General y Aplicada, 54(4), 635-657.

Recuperado de https://goo.gl/5KyEf5

Watson, D. \& Tellegen, A. (1985). Toward a consensual structure of mood. Psychological Bulletin, 98(2), 219-235.

http://dx.doi.org/10.1037/0033-2909.98.2.219

Weinberg, A. \& Hajcak, G. (2010). Beyond good and evil: The time-course of neural activity elicited by specific picture content. Emotion, 10(6), 767-782. http://dx.doi.org/10.1037/a0020242

Wundt, W. (1896). Outlines of Psychology. New York, New York: Sterchert.

Fecha de recepción: 2 de noviembre de 2016. Fecha de aceptación: 7 de diciembre de 2017. 
Anexo

Evaluaciones de las 60 imágenes que conforman el IAPS

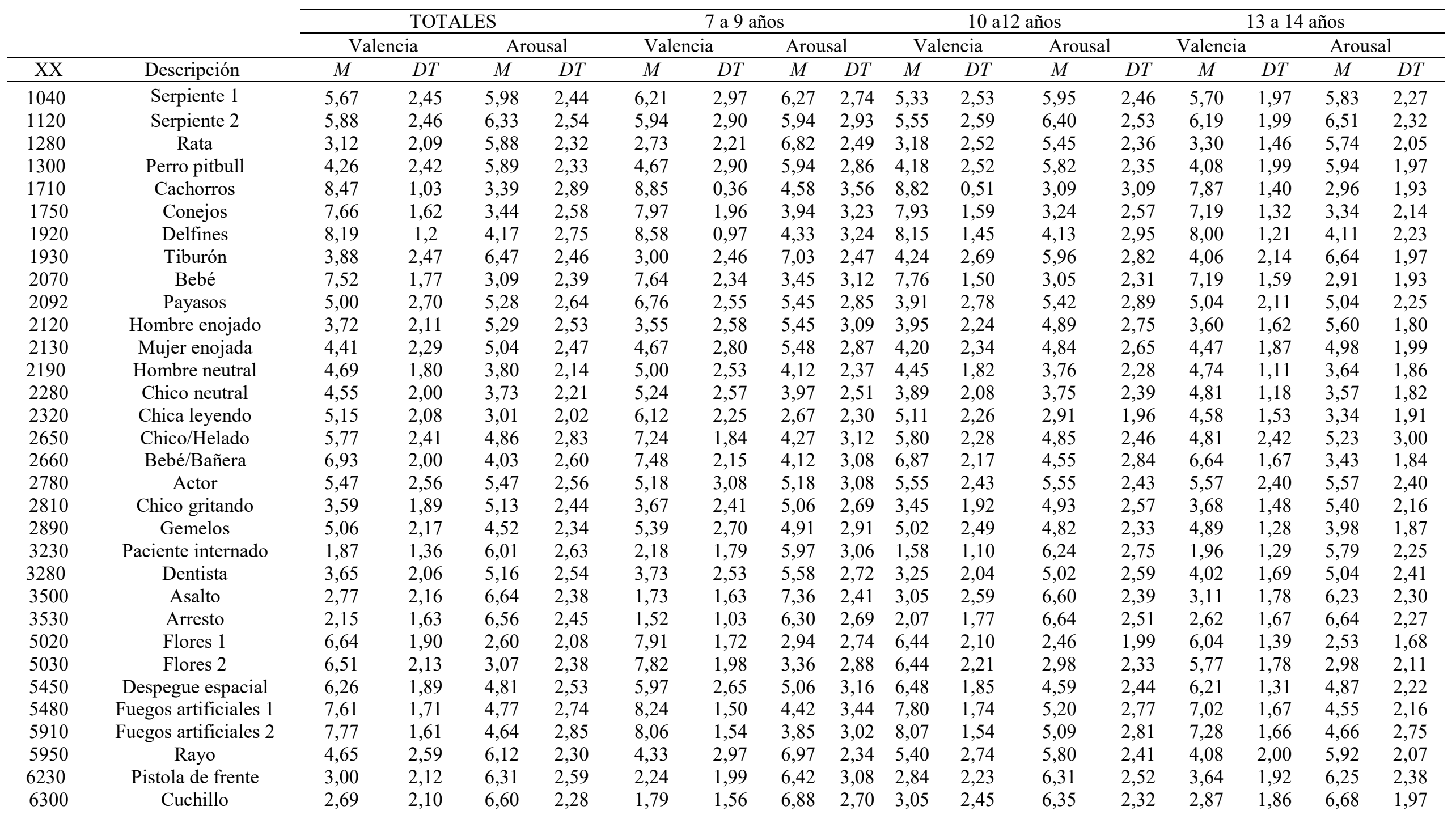




\begin{tabular}{|c|c|c|c|c|c|c|c|c|c|c|c|c|c|c|c|c|c|}
\hline 6370 & Hombre con máscara & 3,41 & 2,21 & 5,79 & 2,40 & 3,82 & 2,73 & 5,06 & 2,67 & 3,27 & 2,35 & 6,07 & 2,56 & 3,30 & 1,64 & 5,96 & 1,98 \\
\hline 7000 & Palo de amasar & 4,99 & 1,78 & 3,23 & 2,16 & 5,73 & 2,44 & 2,91 & 2,35 & 4,62 & 1,73 & 3,20 & 2,17 & 4,92 & 1,14 & 3,45 & 2,05 \\
\hline 7010 & Canasta & 4,98 & 1,92 & 3,16 & 2,17 & 5,52 & 2,35 & 3,48 & 2,49 & 4,76 & 1,95 & 2,93 & 2,11 & 4,87 & 1,56 & 3,19 & 2,06 \\
\hline 7030 & Plancha & 4,93 & 1,90 & 3,45 & 2,27 & 5,27 & 2,47 & 3,52 & 2,71 & 4,84 & 1,95 & 3,40 & 2,25 & 4,81 & 1,40 & 3,47 & 2,04 \\
\hline 7040 & Pala & 4,74 & 1,82 & 3,09 & 2,10 & 4,94 & 2,46 & 3,27 & 2,63 & 4,53 & 2,04 & 2,96 & 2,07 & 4,83 & 0,91 & 3,11 & 1,79 \\
\hline 7080 & Tenedor & 5,14 & 1,84 & 3,31 & 2,14 & 5,58 & 2,54 & 3,76 & 2,85 & 4,94 & 1,87 & 3,37 & 2,08 & 5,06 & 1,17 & 2,98 & 1,64 \\
\hline 7090 & Libro & 5,26 & 2,28 & 3,19 & 2,43 & 6,12 & 2,47 & 3,45 & 2,98 & 5,24 & 2,40 & 2,82 & 2,20 & 4,74 & 1,89 & 3,42 & 2,27 \\
\hline 7100 & Hidrante & 5,23 & 1,98 & 3,27 & 2,26 & 5,36 & 2,54 & 3,61 & 2,73 & 5,07 & 2,06 & 3,27 & 2,32 & 5,32 & 1,48 & 3,06 & 1,87 \\
\hline 7130 & Camión & 4,85 & 1,79 & 3,60 & 2,25 & 5,00 & 2,28 & 3,33 & 2,45 & 5,05 & 2,02 & 3,65 & 2,62 & 4,55 & 1,05 & 3,72 & 1,68 \\
\hline 7150 & Paraguas & 5,11 & 1,72 & 3,26 & 2,16 & 6,00 & 2,32 & 3,33 & 2,75 & 4,76 & 1,60 & 3,16 & 2,13 & 4,91 & 1,17 & 3,32 & 1,81 \\
\hline 7170 & Lámpara & 5,13 & 1,97 & 3,18 & 2,22 & 5,70 & 2,47 & 3,21 & 2,64 & 4,71 & 2,12 & 3,18 & 2,38 & 5,23 & 1,31 & 3,15 & 1,76 \\
\hline 7250 & Torta de cumpleaños & 6,23 & 2,33 & 3,80 & 2,32 & 7,06 & 2,36 & 3,82 & 2,86 & 5,98 & 2,45 & 3,91 & 2,28 & 5,96 & 2,10 & 3,68 & 2,02 \\
\hline 7330 & Helado & 7,58 & 1,84 & 4,32 & 2,75 & 7,88 & 1,64 & 5,15 & 3,23 & 8,02 & 1,86 & 4,11 & 3,01 & 6,94 & 1,81 & 4,02 & 2,01 \\
\hline 7380 & Pizza/Cucarachas & 2,97 & 2,28 & 5,43 & 2,54 & 2,97 & 2,78 & 5,88 & 2,85 & 2,71 & 2,18 & 5,42 & 2,49 & 3,25 & 2,06 & 5,15 & 2,41 \\
\hline 7390 & Helado de agua & 6,81 & 2,29 & 3,67 & 2,50 & 7,67 & 2,01 & 3,55 & 3,12 & 6,09 & 2,72 & 4,04 & 2,52 & 7,02 & 1,73 & 3,38 & 2,03 \\
\hline 7400 & Chocolate & 7,72 & 1,73 & 4,21 & 2,83 & 7,85 & 1,48 & 4,55 & 3,12 & 7,85 & 1,96 & 4,71 & 3,11 & 7,51 & 1,64 & 3,47 & 2,16 \\
\hline 7410 & Bolas de chocolate & 7,37 & 1,88 & 3,99 & 2,79 & 7,79 & 1,58 & 4,00 & 3,16 & 7,51 & 2,12 & 4,31 & 3,05 & 6,96 & 1,75 & 3,64 & 2,22 \\
\hline 7430 & Chocolate relleno & 6,68 & 2,30 & 3,96 & 2,47 & 6,64 & 2,70 & 4,15 & 2,76 & 6,85 & 2,48 & 4,25 & 2,70 & 6,53 & 1,85 & 3,55 & 1,98 \\
\hline 7510 & Edificio & 6,52 & 2,04 & 3,54 & 2,41 & 6,67 & 2,58 & 3,30 & 2,71 & 6,60 & 2,00 & 3,96 & 2,59 & 6,34 & 1,73 & 3,25 & 1,96 \\
\hline 8260 & Motociclista & 6,26 & 2,38 & 4,71 & 2,72 & 6,70 & 2,76 & 4,36 & 2,99 & 6,45 & 2,25 & 4,36 & 2,73 & 5,77 & 2,22 & 5,28 & 2,48 \\
\hline 8490 & Montaña Rusa & 6,64 & 2,11 & 5,19 & 2,69 & 6,36 & 2,71 & 4,88 & 2,92 & 6,58 & 2,11 & 5,11 & 2,77 & 6,87 & 1,67 & 5,47 & 2,49 \\
\hline 8510 & Auto deportivo & 6,96 & 2,04 & 4,55 & 2,66 & 7,55 & 2,14 & 5,09 & 3,22 & 7,11 & 2,07 & 4,38 & 2,63 & 6,45 & 1,88 & 4,40 & 2,30 \\
\hline 8620 & Caballo en circo & 6,19 & 2,29 & 4,13 & 2,36 & 7,58 & 1,92 & 3,79 & 2,63 & 5,85 & 2,44 & 4,18 & 2,40 & 5,68 & 2,04 & 4,30 & 2,17 \\
\hline 9050 & Accidente aéreo & 2,77 & 2,13 & 6,06 & 2,51 & 3,64 & 2,66 & 5,76 & 2,75 & 2,51 & 2,20 & 5,87 & 2,63 & 2,51 & 1,51 & 6,45 & 2,22 \\
\hline 9403 & Soldado & 3,81 & 2,46 & 5,67 & 2,39 & 4,30 & 3,02 & 5,33 & 2,80 & 3,64 & 2,44 & 5,45 & 2,33 & 3,68 & 2,09 & 6,09 & 2,15 \\
\hline 9421 & Soldado herido & 2,73 & 1,98 & 5,68 & 2,58 & 2,42 & 2,35 & 6,55 & 2,73 & 2,60 & 1,81 & 5,04 & 2,67 & 3,06 & 1,90 & 5,81 & 2,25 \\
\hline 9480 & Calavera & 3,51 & 2,40 & 6,12 & 2,35 & 3,88 & 2,79 & 5,67 & 2,48 & 3,55 & 2,60 & 6,36 & 2,54 & 3,25 & 1,91 & 6,15 & 2,05 \\
\hline 9925 & Fuego & 3,44 & 2,303 & 6,04 & 2,53 & 3,52 & 2,58 & 5,94 & 2,68 & 3,69 & 2,51 & 5,91 & 2,60 & 3,13 & 1,87 & 6,23 & 2,41 \\
\hline
\end{tabular}

Notas. Media y desviación típica en las dimensiones de valencia y activación para el subconjunto del IAPS adaptado a niños para el total de los sujetos ( $\mathrm{n}=141$ ), para el subgrupo de 7 a 9 años $(n=33)$, para el subgrupo de 10 a 12 años $(n=55)$ y para el subgrupo de 13 a 14 años $(n=53)$. 